\title{
Extremal Unimodular Lattices in Dimension 36
}

\begin{abstract}
Masaaki Harada
Research Center for Pure and Applied Mathematics, Graduate School of Information Sciences, Tohoku University, Sendai 980-8579, Japan
\end{abstract}

Correspondence should be addressed to Masaaki Harada; mharada@m.tohoku.ac.jp

Received 15 August 2014; Accepted 6 November 2014; Published 30 November 2014

Academic Editor: Jun-Ming Xu

Copyright (C) 2014 Masaaki Harada. This is an open access article distributed under the Creative Commons Attribution License, which permits unrestricted use, distribution, and reproduction in any medium, provided the original work is properly cited.

New extremal odd unimodular lattices in dimension 36 are constructed. Some new odd unimodular lattices in dimension 36 with long shadows are also constructed.

"Dedicated to Professor Vladimir D. Tonchev on His 60th Birthday"

\section{Introduction}

A (Euclidean) lattice $L \subset \mathbb{R}^{n}$ in dimension $n$ is unimodular if $L=L^{*}$, where the dual lattice $L^{*}$ of $L$ is defined as $\left\{x \in \mathbb{R}^{n} \mid\right.$ $(x, y) \in \mathbb{Z}$ for all $y \in L\}$ under the standard inner product $(x, y)$. A unimodular lattice is called even if the norm $(x, x)$ of every vector $x$ is even. A unimodular lattice, which is not even, is called odd. An even unimodular lattice in dimension $n$ exists if and only if $n \equiv 0(\bmod 8)$, while an odd unimodular lattice exists for every dimension. Two lattices $L$ and $L^{\prime}$ are isomorphic, denoted by $L \cong L^{\prime}$, if there exists an orthogonal matrix $A$ with $L^{\prime}=L \cdot A$, where $L \cdot A=\{x A \mid x \in L\}$. The automorphism group $\operatorname{Aut}(L)$ of $L$ is the group of all orthogonal matrices $A$ with $L=L \cdot A$.

Rains and Sloane [6] showed that the minimum norm $\min (L)$ of a unimodular lattice $L$ in dimension $n$ is bounded by $\min (L) \leq 2\lfloor n / 24\rfloor+2$ unless $n=23$ when $\min (L) \leq 3$. We say that a unimodular lattice meeting the upper bound is extremal.

The smallest dimension for which there is an odd unimodular lattice with minimum norm (at least) 4 is 32 (see [1]). There are exactly five odd unimodular lattices in dimension 32 having minimum norm 4, up to isomorphism [7]. For dimensions 33,34 , and 35 , the minimum norm of an odd unimodular lattice is at most 3 (see [1]). The next dimension for which there is an odd unimodular lattice with minimum norm (at least) 4 is 36 . Four extremal odd unimodular lattices in dimension 36 are known, namely, Sp4(4)D8.4 in [1], $G_{36}$ in [2, Table 2], $N_{36}$ in [3, Section 3], and $A_{4}\left(C_{36}\right)$ in [4, Section 3]. Recently, one more lattice has been found, namely, $A_{6}\left(C_{36,6}\left(D_{18}\right)\right)$ in [5, Table II]. This situation motivates us to improve the number of known nonisomorphic extremal odd unimodular lattices in dimension 36. The main aim of this paper is to prove the following.

Proposition 1. There are at least 26 nonisomorphic extremal odd unimodular lattices in dimension 36.

The above proposition is established by constructing new extremal odd unimodular lattices in dimension 36 from selfdual $\mathbb{Z}_{k}$-codes, where $\mathbb{Z}_{k}$ is the ring of integers modulo $k$, by using two approaches. One approach is to consider self-dual $\mathbb{Z}_{4}$-codes. Let $B$ be a binary doubly even code of length 36 satisfying the following conditions:

the minimum weight of $B$ is at least 16 ,

the minimum weight of its dual code $B^{\perp}$ is at least 4 .

Then a self-dual $\mathbb{Z}_{4}$-code with residue code $B$ gives anextremal odd unimodular lattice in dimension 36 by Construction A. We show that a binary doubly even $[36,7]$ code satisfying conditions (1) has weight enumerator $1+63 y^{16}+$ $63 y^{20}+y^{36}$ (Lemma 2). It was shown in [8] that there are four codes having the weight enumerator, up to equivalence. 
We construct ten new extremal odd unimodular lattices in dimension 36 from self-dual $\mathbb{Z}_{4}$-codes whose residue codes are doubly even $[36,7]$ codes satisfying conditions (1) (Lemma 4). New odd unimodular lattices in dimension 36 with minimum norm 3 having shadows of minimum norm 5 are constructed from some of the new lattices (Proposition 7). These are often called unimodular lattices with long shadows (see [9]). The other approach is to consider self-dual $\mathbb{Z}_{k^{-}}$ codes $(k=5,6,7,9,19)$, which have generator matrices of a special form given in (14). Eleven more new extremal odd unimodular lattices in dimension 36 are constructed by Construction A (Lemma 8). Finally, we give a certain short observation on ternary self-dual codes related to extremal odd unimodular lattices in dimension 36.

All computer calculations in this paper were done by Magma [10].

\section{Preliminaries}

2.1. Unimodular Lattices. Let $L$ be an odd unimodular lattice and let $L_{0}$ denote the even sublattice, that is, the sublattice of vectors of even norms. Then $L_{0}$ is a sublattice of $L$ of index 2 [7]. The shadow $S(L)$ of $L$ is defined to be $L_{0}^{*} \backslash L$. There are cosets $L_{1}, L_{2}, L_{3}$ of $L_{0}$ such that $L_{0}^{*}=L_{0} \cup L_{1} \cup L_{2} \cup L_{3}$, where $L=L_{0} \cup L_{2}$ and $S=L_{1} \cup L_{3}$. Shadows for odd unimodular lattices appeared in [7] and also in [11, p. 440], in order to provide restrictions on the theta series of odd unimodular lattices. Two lattices $L$ and $L^{\prime}$ are neighbors if both lattices contain a sublattice of index 2 in common. If $L$ is an odd unimodular lattice in dimension divisible by 4 , then there are two unimodular lattices containing $L_{0}$, which are rather $L$, namely, $L_{0} \cup L_{1}$ and $L_{0} \cup L_{3}$. Throughout this paper, we denote the two unimodular neighbors by

$$
N e_{1}(L)=L_{0} \cup L_{1}, \quad N e_{2}(L)=L_{0} \cup L_{3} .
$$

The theta series $\theta_{L}(q)$ of $L$ is the formal power series $\theta_{L}(q)=\sum_{x \in L} q^{(x, x)}$. The kissing number of $L$ is the second nonzero coefficient of the theta series of $L$, that is, the number of vectors of minimum norm in $L$. Conway and Sloane [7] gave some characterization of theta series of odd unimodular lattices and their shadows. Using $[7,(2),(3)]$, it is easy to determine the possible theta series $\theta_{L_{36}}(q)$ and $\theta_{S\left(L_{36}\right)}(q)$ of an extremal odd unimodular lattice $L_{36}$ in dimension 36 and its shadow $S\left(L_{36}\right)$ :

$$
\begin{aligned}
\theta_{L_{36}}(q)= & 1+(42840+4096 \alpha) q^{4} \\
& +(1916928-98304 \alpha) q^{5}+\cdots, \\
\theta_{S\left(L_{36}\right)}(q)= & \alpha q+(960-60 \alpha) q^{3} \\
& +(3799296+1734 \alpha) q^{5}+\cdots,
\end{aligned}
$$

respectively, where $\alpha$ is a nonnegative integer. It follows from the coefficients of $q$ and $q^{3}$ in $\theta_{S\left(L_{36}\right)}(q)$ that $0 \leq \alpha \leq 16$.

2.2. Self-Dual $\mathbb{Z}_{k}$-Codes and Construction $A$. Let $\mathbb{Z}_{k}$ be the ring of integers modulo $k$, where $k$ is a positive integer greater than 1. A $\mathbb{Z}_{k}$-code $C$ of length $n$ is a $\mathbb{Z}_{k}$-submodule of $\mathbb{Z}_{k}^{n}$. Two $\mathbb{Z}_{k}$-codes are equivalent if one can be obtained from the other by permuting the coordinates and (if necessary) changing the signs of certain coordinates. A code $C$ is self-dual if $C=C^{\perp}$, where the dual code $C^{\perp}$ of $C$ is defined as $\left\{x \in \mathbb{Z}_{k}^{n} \mid x \cdot y=0\right.$ for all $y \in C\}$, under the standard inner product $x \cdot y$.

If $C$ is a self-dual $\mathbb{Z}_{k}$-code of length $n$, then the lattice

$$
\begin{aligned}
& A_{k}(C) \\
& \quad=\frac{1}{\sqrt{k}}\left\{\left(x_{1}, \ldots, x_{n}\right) \in \mathbb{Z}^{n}\left(x_{1} \bmod k, \ldots, x_{n} \bmod k\right) \in C\right\}
\end{aligned}
$$

is a unimodular lattice in dimension $n$. This construction of lattices is called Construction A.

\section{From Self-Dual $\mathbb{Z}_{4}$-Codes}

From now on, we omit the term "odd" for odd unimodular lattices in dimension 36 , since all unimodular lattices in dimension 36 are odd. In this section, we construct ten new nonisomorphic extremal unimodular lattices in dimension 36 from self-dual $\mathbb{Z}_{4}$-codes by Construction A. Five new nonisomorphic unimodular lattices in dimension 36 with minimum norm 3 having shadows of minimum norm 5 are also constructed.

3.1. Extremal Unimodular Lattices. Every $\mathbb{Z}_{4}$-code $C$ of length $n$ has two binary codes $C^{(1)}$ and $C^{(2)}$ associated with $C$ :

$$
\begin{aligned}
& C^{(1)}=\{c \bmod 2 \mid c \in C\}, \\
& C^{(2)}=\left\{c \bmod 2 \mid c \in \mathbb{Z}_{4}^{n}, 2 c \in C\right\} .
\end{aligned}
$$

The binary codes $C^{(1)}$ and $C^{(2)}$ are called the residue and torsion codes of $C$, respectively. If $C$ is a self-dual $\mathbb{Z}_{4}$-code, then $C^{(1)}$ is a binary doubly even code with $C^{(2)}=C^{(1)^{\perp}}[12]$. Conversely, starting from a given binary doubly even code $B$, a method for construction of all self-dual $\mathbb{Z}_{4}$-codes $C$ with $C^{(1)}=B$ was given in $[13$, Section 3$]$.

The Euclidean weight of a codeword $x=\left(x_{1}, \ldots, x_{n}\right)$ of $C$ is $m_{1}(x)+4 m_{2}(x)+m_{3}(x)$, where $m_{\alpha}(x)$ denotes the number of components $i$ with $x_{i}=\alpha(\alpha=1,2,3)$. The minimum Euclidean weight $d_{E}(C)$ of $C$ is the smallest Euclidean weight among all nonzero codewords of $C$. It is easy to see that $\min \left\{d\left(C^{(1)}\right), 4 d\left(C^{(2)}\right)\right\} \leq d_{E}(C)$, where $d\left(C^{(i)}\right)$ denotes the minimum weight of $C^{(i)}(i=1,2)$. In addition, $d_{E}(C) \leq$ $4 d\left(C^{(2)}\right)$ and $A_{4}(C)$ has minimum norm $\min \left\{4, d_{E}(C) / 4\right\}$ (see, e.g., [3]). Hence, if there is a binary doubly even code $B$ of length 36 satisfying conditions (1), then an extremal unimodular lattice in dimension 36 is constructed as $A_{4}(C)$, through a self-dual $\mathbb{Z}_{4}$-code $C$ with $C^{(1)}=B$. If there is a binary $[36, k]$ code $B$ satisfying conditions (1), then $k=7$ or 8 (see [14]).

Lemma 2. Let $B$ be a binary doubly even $[36,7]$ code satisfying conditions (1). Then the weight enumerator of $B$ is $1+63 y^{16}+$ $63 y^{20}+y^{36}$. 
Proof. The weight enumerator of $B$ is written as

$$
\begin{aligned}
W_{B}(y)= & 1+a y^{16}+b y^{20}+c y^{24}+d y^{28} \\
& +e y^{32}+\left(2^{7}-1-a-b-c-d-e\right) y^{36}
\end{aligned}
$$

where $a, b, c, d$, and $e$ are nonnegative integers. By the MacWilliams identity, the weight enumerator of $B^{\perp}$ is given by

$$
\begin{aligned}
W_{B^{\perp}}(y)= & +\frac{1}{16}(-567+5 a+4 b+3 c+2 d+e) y \\
& +\frac{1}{2}(1260-10 a-10 b-9 c-7 d-4 e) y^{2} \\
& +\frac{1}{16}(-112455+885 a+900 b \\
& +883 c+770 d+497 e) y^{3}+\cdots
\end{aligned}
$$

Since $d\left(B^{\perp}\right) \geq 4$, the weight enumerator of $B$ is written using $a$ and $b$ :

$$
\begin{aligned}
W_{B}(y)= & 1+a y^{16}+b y^{20}+(882-10 a-4 b) y^{24} \\
& +(-1638+20 a+6 b) y^{28} \\
& +(1197-15 a-4 b) y^{32} \\
& +(-314+4 a+b) y^{36}
\end{aligned}
$$

Suppose that $B$ does not contain the all-one vector 1 . Then $b=314-4 a$. In this case, since the coefficients of $y^{24}$ and $y^{28}$ are $-374+6 a$ and $246-4 a$, these yield that $a \geq 62$ and $a \leq$ 61 , respectively, which gives the contradiction. Hence, $B$ contains 1 . Then $b=315-4 a$. Since the coefficient $a-63$ of $y^{32}$ is 0 , the weight enumerator of $B$ is uniquely determined as $1+63 y^{16}+63 y^{20}+y^{36}$.

Remark 3. A similar approach shows that the weight enumerator of a binary doubly even $[36,8]$ code $B$ satisfying conditions (1) is uniquely determined as $1+153 y^{16}+72 y^{20}+$ $30 y^{24}$.

It was shown in [8] that there are four inequivalent binary $[36,7,16]$ codes containing 1 . The four codes are doubly even. Hence, there are exactly four binary doubly even [36, 7] codes satisfying conditions (1), up to equivalence. The four codes are optimal in the sense that these codes achieve the Gray-Rankin bound, and the codewords of weight 16 are corresponding to quasi-symmetric SDP 2- $(36,16,12)$ designs [15]. Let $B_{36, i}$ be the binary doubly even $[36,7,16]$ code corresponding to the quasi-symmetric SDP $2-(36,16,12)$ design, which is the residual design of the symmetric SDP 2-(64,28, 12) design $D_{i}$ in [8, Section 5] $(i=1,2,3,4)$. As described above, all selfdual $\mathbb{Z}_{4}$-codes $C$ with $C^{(1)}=B_{36, i}$ have $d_{E}(C)=16(i=$ $1,2,3,4)$. Hence, $A_{4}(C)$ are extremal.

Using the method in [13, Section 3], self-dual $\mathbb{Z}_{4}$-codes $C$ are constructed from $B_{36, i}$. Then ten extremal unimodular lattices $A_{4}\left(C_{36, i}\right)(i=1,2, \ldots, 10)$ are constructed, where
$C_{36, i}^{(1)}=B_{36,2}(i=1,2,3), C_{36, i}^{(1)}=B_{36,3}(i=4,5,6,7)$, and $C_{36, i}^{(1)}=B_{36,4}(i=8,9,10)$. To distinguish between the known lattices and our lattices, we give in Table 1 the kissing numbers $\tau(L),\left\{n_{1}(L), n_{2}(L)\right\}$, and the orders \# Aut $(L)$ of the automorphism groups, where $n_{i}(L)$ denotes the number of vectors of norm 3 in $N e_{i}(L)$ defined in $(2)(i=1,2)$. These have been calculated by Magma. Table 1 shows the following.

Lemma 4. The five known lattices and the ten extremal unimodular lattices $A_{4}\left(C_{36, i}\right)(i=1,2, \ldots, 10)$ are nonisomorphic to each other.

Remark 5. In this way, we have found two more extremal unimodular lattices $A_{4}(C)$, where $C$ are self-dual $\mathbb{Z}_{4}$-codes with $C^{(1)}=B_{36,1}$. However, we have verified by MAgma that the two lattices are isomorphic to $N_{36}$ in [3] and $A_{4}\left(C_{36}\right)$ in [4].

Remark 6. For $L=A_{4}\left(C_{36, i}\right)(i=1,2, \ldots, 10)$, it follows from $\tau(L)$ and $\left\{n_{1}(L), n_{2}(L)\right\}$ that one of the two unimodular neighbors $\mathrm{Ne}_{1}(L)$ and $\mathrm{Ne}_{2}(L)$ defined in (2) is extremal. We have verified by MAGMA that the extremal one is isomorphic to $A_{4}\left(C_{36, i}\right)$.

For $i=1,2, \ldots, 10$, the code $C_{36, i}$ is equivalent to some code $\overline{C_{36, i}}$ with generator matrix of the form

$$
\left(\begin{array}{ccc}
I_{7} & A & B_{1}+2 B_{2} \\
O & 2 I_{22} & 2 D
\end{array}\right)
$$

where $A, B_{1}, B_{2}$, and $D$ are $(1,0)$-matrices, $I_{n}$ denotes the identity matrix of order $n$, and $O$ denotes the $22 \times 7$ zero matrix. We only list in (11) the $7 \times 29$ matrix $M_{i}=$ $\left(\begin{array}{ll}A & B_{1}+2 B_{2}\end{array}\right)$ to save space. Note that $\left(\begin{array}{lll}O & 2 I_{22} & 2 D\end{array}\right)$ in (10) can be obtained from $\left(I_{7} A B_{1}+2 B_{2}\right)$ since ${\overline{C_{36, i}}}^{(2)}=$ ${\overline{C_{36, i}}}^{(1)^{\perp}}$. A generator matrix of $A_{4}\left(C_{36, i}\right)$ is obtained from that of $C_{36, i}$

$$
\begin{aligned}
M_{1}= & \left(\begin{array}{l}
01101111111000000110000333322 \\
10101101001000111110011210223 \\
11000011001110100111100010321 \\
11010100101011010011012032011 \\
00110101010101001101013312302 \\
00000000000001111111112111333 \\
00011111111110000001111213133
\end{array}\right), \\
M_{2}= & \left(\begin{array}{l}
01101111111000000110002111100 \\
10101101001000111110011210023 \\
11000011001110100111100010123 \\
11010100101011010011012032211 \\
00110101010101001101013312300 \\
00000000000001111111112113131 \\
0001111111110000001111211331
\end{array}\right),
\end{aligned}
$$


TABLE 1: Extremal unimodular lattices in dimension 36.

\begin{tabular}{|c|c|c|c|}
\hline Lattices $L$ & $\tau(L)$ & $\left\{n_{1}(L), n_{2}(L)\right\}$ & \# Aut $(L)$ \\
\hline Sp4(4)D8.4 in [1] & 42840 & $\{480,480\}$ & 31334400 \\
\hline$G_{36}$ in [2, Table 2] & 42840 & $\{144,816\}$ & 576 \\
\hline$N_{36}$ in $[3]$ & 42840 & $\{0,960\}$ & 849346560 \\
\hline$A_{4}\left(C_{36}\right)$ in $[4]$ & 51032 & $\{0,840\}$ & 660602880 \\
\hline$A_{6}\left(C_{36,6}\left(D_{18}\right)\right)$ in $[5]$ & 42840 & $\{384,576\}$ & 288 \\
\hline$A_{4}\left(C_{36,1}\right)$ in Section 3 & 51032 & $\{0,840\}$ & 6291456 \\
\hline$A_{4}\left(C_{36,2}\right)$ in Section 3 & 42840 & $\{0,960\}$ & 6291456 \\
\hline$A_{4}\left(C_{36,3}\right)$ in Section 3 & 51032 & $\{0,840\}$ & 22020096 \\
\hline$A_{4}\left(C_{36,4}\right)$ in Section 3 & 51032 & $\{0,840\}$ & 1966080 \\
\hline$A_{4}\left(C_{36,5}\right)$ in Section 3 & 51032 & $\{0,840\}$ & 1572864 \\
\hline$A_{4}\left(C_{36,6}\right)$ in Section 3 & 42840 & $\{0,960\}$ & 2621440 \\
\hline$A_{4}\left(C_{36,7}\right)$ in Section 3 & 42840 & $\{0,960\}$ & 1966080 \\
\hline$A_{4}\left(C_{36,8}\right)$ in Section 3 & 42840 & $\{0,960\}$ & 393216 \\
\hline$A_{4}\left(C_{36,9}\right)$ in Section 3 & 51032 & $\{0,840\}$ & 1376256 \\
\hline$A_{4}\left(C_{36,10}\right)$ in Section 3 & 51032 & $\{0,840\}$ & 393216 \\
\hline$A_{5}\left(D_{36,1}\right)$ in Section 4 & 42840 & $\{144,816\}$ & 144 \\
\hline$A_{5}\left(D_{36,2}\right)$ in Section 4 & 42840 & $\{456,504\}$ & 72 \\
\hline$A_{6}\left(D_{36,3}\right)$ in Section 4 & 42840 & $\{240,720\}$ & 288 \\
\hline$A_{6}\left(D_{36,4}\right)$ in Section 4 & 42840 & $\{240,720\}$ & 576 \\
\hline$A_{7}\left(D_{36,5}\right)$ in Section 4 & 42840 & $\{288,672\}$ & 288 \\
\hline$A_{7}\left(D_{36,6}\right)$ in Section 4 & 42840 & $\{144,816\}$ & 72 \\
\hline$A_{7}\left(D_{36,7}\right)$ in Section 4 & 42840 & $\{144,816\}$ & 288 \\
\hline$A_{9}\left(D_{36,8}\right)$ in Section 4 & 42840 & $\{384,576\}$ & 144 \\
\hline$A_{19}\left(D_{36,9}\right)$ in Section 4 & 42840 & $\{288,672\}$ & 144 \\
\hline$A_{5}\left(E_{36,1}\right)$ in Section 4 & 42840 & $\{456,504\}$ & 72 \\
\hline$A_{6}\left(E_{36,2}\right)$ in Section 4 & 42840 & $\{384,576\}$ & 144 \\
\hline
\end{tabular}

$$
\begin{aligned}
M_{3}= & \left(\begin{array}{l}
01101111111000000110002131300 \\
10101101001000111110011210223 \\
11000011001110100111100010121 \\
11010100101011010011012032011 \\
00110101010101001101013310102 \\
00000000000001111111112131131 \\
00011111111110000001111233331
\end{array}\right) \\
M_{4}= & \left(\begin{array}{l}
01101111111001111101100220313 \\
10101101001001001110101231032 \\
11000011001110110100102101101 \\
00111001100011100101113010223 \\
11011000011101100011100021110 \\
00000000000001111111112111131 \\
00011111111110000001113301311
\end{array}\right) \\
M_{5}= & \left(\begin{array}{l}
01101111111001111101102202133 \\
10101101001001001110101231032 \\
11000011001110110100102101101 \\
00111001100011100101113010223 \\
11011000011101100011100221310 \\
0000000000000111111112111131 \\
0001111111110000001113301311
\end{array}\right)
\end{aligned}
$$

$$
M_{6}=\left(\begin{array}{l}
01101111111001111101102000313 \\
10101101001001001110101231032 \\
11000011001110110100102101103 \\
00111001100011100101113210223 \\
11011000011101100011100021110 \\
0000000000000111111112313113 \\
00011111111110000001113103333
\end{array}\right)
$$$$
M_{7}=\left(\begin{array}{l}
01101111111001111101102202313 \\
10101101001001001110101231030 \\
11000011001110110100102101121 \\
00111001100011100101113210201 \\
11011000011101100011100021112 \\
0000000000000111111112111113 \\
00011111111110000001113301333
\end{array}\right)
$$$$
M_{8}=\left(\begin{array}{l}
11111110000111111111000022313 \\
11110001100111000000001130313 \\
01000110111001110100011231021 \\
10011100001011100001101233011 \\
01010101010010101101003301321 \\
000000000111111111111233111 \\
01111111100000000111111233313
\end{array}\right) \text {, }
$$ 


$$
\begin{aligned}
& M_{9}=\left(\begin{array}{l}
11111110000111111111002200111 \\
11110001100111000000001332311 \\
01000110111001110100013211021 \\
10011100001011100001103213011 \\
01010101010010101101000331321 \\
01111111100000000111111211113 \\
00000000011111111111111303313
\end{array}\right), \\
& M_{10}=\left(\begin{array}{l}
11111110000111111111002202313 \\
11110001100111000000001130311 \\
01000110111001110100011211021 \\
10011100001011100001101213011 \\
01010101010010101101003321123 \\
0000000001111111111112313313 \\
0111111110000000011111211111
\end{array}\right) .
\end{aligned}
$$

3.2. Unimodular Lattices with Long Shadows. The possible theta series of a unimodular lattice $L$ in dimension 36 having minimum norm 3 and its shadow are as follows:

$$
\begin{gathered}
1+(960-\alpha) q^{3}+(42840+4096 \beta) q^{4}+\cdots, \\
\beta q+(\alpha-60 \beta) q^{3}+(3833856-36 \alpha+1734 \beta) q^{5}+\cdots,
\end{gathered}
$$

respectively, where $\alpha$ and $\beta$ are integers with $0 \leq \beta \leq \alpha / 60<$ 16 [3]. Then the kissing number of $L$ is at most 960 and $\min (S(L)) \leq 5$. Unimodular lattices $L$ with $\min (L)=3$ and $\min (S(L))=5$ are often called unimodular lattices with long shadows (see [9]). Only one unimodular lattice $L$ in dimension 36 with $\min (L)=3$ and $\min (S(L))=5$ was known, namely, $A_{4}\left(C_{36}\right)$ in [3].

Let $L$ be one of $A_{4}\left(C_{36,2}\right), A_{4}\left(C_{36,6}\right), A_{4}\left(C_{36,7}\right)$, and $A_{4}\left(C_{36,8}\right)$. Since $\left\{n_{1}(L), n_{2}(L)\right\}=\{0,960\}$, one of the two unimodular neighbors $\mathrm{Ne}_{1}(L)$ and $\mathrm{Ne}_{2}(L)$ in (2) is extremal and the other is a unimodular lattice $L^{\prime}$ with minimum norm 3 having shadow of minimum norm 5 . We denote such lattices $L^{\prime}$ constructed from $A_{4}\left(C_{36,2}\right), A_{4}\left(C_{36,6}\right), A_{4}\left(C_{36,7}\right)$, and $A_{4}\left(C_{36,8}\right)$ by $N_{36,1}, N_{36,2}, N_{36,3}$, and $N_{36,4}$, respectively. We list in Table 2 the orders \# Aut $\left(N_{36, i}\right)(i=1,2,3,4)$ of the automorphism groups, which have been calculated by MaGMA. Table 2 shows the following.

Proposition 7. There are at least 5 nonisomorphic unimodular lattices $L$ in dimension 36 with $\min (L)=3$ and $\min (S(L))=5$.

\section{From Self-Dual $\mathbb{Z}_{k}$-Codes $(k \geq 5)$}

In this section, we construct more extremal unimodular lattices in dimension 36 from self-dual $\mathbb{Z}_{k}$-codes $(k \geq 5)$.

Let $A^{T}$ denote the transpose of a matrix $A$. An $n \times n$ matrix is negacirculant if it has the following form:

$$
\left(\begin{array}{cccc}
r_{0} & r_{1} & \cdots & r_{n-1} \\
-r_{n-1} & r_{0} & \cdots & r_{n-2} \\
-r_{n-2} & -r_{n-1} & \cdots & r_{n-3} \\
\vdots & \vdots & & \vdots \\
-r_{1} & -r_{2} & \cdots & r_{0}
\end{array}\right) .
$$

TABle 2: \# $\operatorname{Aut}\left(N_{36, i}\right)(i=1,2,3,4)$.

\begin{tabular}{lc}
\hline Lattices $L$ & \# Aut $(L)$ \\
\hline$A_{4}\left(C_{36}\right)$ in [3] & 1698693120 \\
$N_{36,1}$ & 12582912 \\
$N_{36,2}$ & 5242880 \\
$N_{36,3}$ & 3932160 \\
$N_{36,4}$ & 786432 \\
\hline
\end{tabular}

Let $D_{36, i}(i=1,2, \ldots, 9)$ and $E_{36, i}(i=1,2)$ be $\mathbb{Z}_{k}$-codes of length 36 with generator matrices of the following form:

$$
\left(\begin{array}{ccc} 
& A & B \\
I_{18} & -B^{T} & A^{T}
\end{array}\right)
$$

where $k$ are listed in Table 3 , and $A$ and $B$ are $9 \times 9$ negacirculant matrices with first rows $r_{A}$ and $r_{B}$ listed in Table 3. It is easy to see that these codes are self-dual since $A A^{T}+B B^{T}=-I_{9}$. Thus, $A_{k}\left(D_{36, i}\right)(i=1,2, \ldots, 9)$ and $A_{k}\left(E_{36, i}\right)(i=1,2)$ are unimodular lattices, for $k$ given in Table 3. In addition, we have verified by MAGMA that these lattices are extremal.

To distinguish between the above eleven lattices and the 15 known lattices, in Table 1 we give $\tau(L),\left\{n_{1}(L), n_{2}(L)\right\}$, and \# $\operatorname{Aut}(L)$, which have been calculated by Magma. The two lattices have the identical $\left(\tau(L),\left\{n_{1}(L), n_{2}(L)\right\}\right.$, \# Aut $(L))$ for each of the pairs $\left(A_{5}\left(E_{36,1}\right), A_{5}\left(D_{36,2}\right)\right)$ and $\left(A_{6}\left(E_{36,2}\right), A_{9}\left(D_{36,8}\right)\right)$. However, we have verified by Magma that the two lattices are nonisomorphic for each pair. Therefore, we have the following.

Lemma 8. The 26 lattices in Table 1 are nonisomorphic to each other.

Lemma 8 establishes Proposition 1.

Remark 9. Similar to Remark 6, it is known [3] that the extremal neighbor is isomorphic to $L$ for the case where $L$ is $N_{36}$ in [3], and we have verified by MAGMA that the extremal neighbor is isomorphic to $L$ for the case where $L$ is $A_{4}\left(C_{36}\right)$ in [4].

\section{Related Ternary Self-Dual Codes}

In this section, we give a certain short observation on ternary self-dual codes related to some extremal odd unimodular lattices in dimension 36 .

5.1. Unimodular Lattices from Ternary Self-Dual Codes. Let $T_{36}$ be a ternary self-dual code of length 36 . The two unimodular neighbors $N e_{1}\left(A_{3}\left(T_{36}\right)\right)$ and $N e_{2}\left(A_{3}\left(T_{36}\right)\right)$ given in (2) are described in [16] as $L_{S}\left(T_{36}\right)$ and $L_{T}\left(T_{36}\right)$. In this section, we use the notation $L_{S}\left(T_{36}\right)$ and $L_{T}\left(T_{36}\right)$, instead of $N e_{1}\left(A_{3}\left(T_{36}\right)\right)$ and $N e_{2}\left(A_{3}\left(T_{36}\right)\right)$, since the explicit constructions and some properties of $L_{S}\left(T_{36}\right)$ and $L_{T}\left(T_{36}\right)$ are given in [16]. By Theorem 6 in [16] (see also Theorem 3.1 in [2]), $L_{T}\left(T_{36}\right)$ is extremal when $T_{36}$ satisfies the following condition 
TABLE 3: Self-dual $\mathbb{Z}_{k}$-codes of length 36 .

\begin{tabular}{lccr}
\hline Codes & $k$ & $r_{A}$ & $r_{B}$ \\
\hline$D_{36,1}$ & 5 & $(0,0,0,1,2,2,0,4,2)$ & $(0,0,0,0,4,3,3,0,1)$ \\
$D_{36,2}$ & 5 & $(0,0,0,1,3,0,2,0,4)$ & $(3,0,4,1,4,0,0,4,4)$ \\
$D_{36,3}$ & 6 & $(0,1,5,3,2,0,3,5,1)$ & $(3,1,0,0,5,1,1,1,1)$ \\
$D_{36,4}$ & 6 & $(0,1,3,5,1,5,5,4,4)$ & $(4,0,3,2,4,5,5,2,4)$ \\
$D_{36,5}$ & $(0,1,6,3,5,0,4,5,4)$ & $(0,1,6,3,5,2,1,5,1)$ \\
$D_{36,6}$ & 7 & $(0,1,1,3,2,6,1,4,6)$ & $(0,1,4,0,5,3,6,2,0)$ \\
$D_{36,7}$ & 7 & $(0,0,0,1,5,5,5,1,1)$ & $(0,5,4,2,5,1,1,3,6)$ \\
$D_{36,8}$ & 7 & $(0,0,0,1,0,4,3,0,0)$ & $(0,4,1,5,3,5,1,7,0)$ \\
$D_{36,9}$ & $(0,0,0,1,15,15,9,6,5)$ & $(14,16,0,14,15,8,8,3,12)$ \\
\hline$E_{36,1}$ & 9 & $(0,1,0,2,1,3,2,2,0)$ & $(2,0,1,0,1,1,2,3,1)$ \\
$E_{36,2}$ & 19 & $(0,1,5,3,4,4,1,1,0)$ & $(4,0,1,3,4,2,3,0,1)$ \\
\hline
\end{tabular}

(a), and both $L_{S}\left(T_{36}\right)$ and $L_{T}\left(T_{36}\right)$ are extremal when $T_{36}$ satisfies the following condition (b):

(a) extremal (minimum weight 12) and admissible (the number of 1's in the components of every codeword of weight 36 is even),

(b) minimum weight 9 and maximum weight 33 .

For each of (a) and (b), no ternary self-dual code satisfying the condition is currently known.

5.2. Condition (a). Suppose that $T_{36}$ satisfies condition (a). Since $T_{36}$ has minimum weight $12, A_{3}\left(T_{36}\right)$ has minimum norm 3 and kissing number 72. By Theorem 6 in [16], $\min \left(L_{T}\left(T_{36}\right)\right)=4$ and $\min \left(L_{S}\left(T_{36}\right)\right)=3$. Hence, since the shadow of $L_{T}\left(T_{36}\right)$ contains no vector of norm 1, by (3) and (4), $L_{T}\left(T_{36}\right)$ has theta series $1+42840 q^{4}+1916928 q^{5}+\cdots$. It follows that $\left\{n_{1}\left(L_{T}\left(T_{36}\right)\right), n_{2}\left(L_{T}\left(T_{36}\right)\right)\right\}=\{72,888\}$.

By Theorem 1 in [17], the possible complete weight enumerator $W_{C}(x, y, z)$ of a ternary extremal self-dual code $C$ of length 36 containing $\mathbf{1}$ is written as

$$
\begin{aligned}
a_{1} \delta_{36} & +a_{2} \alpha_{12}^{3}+a_{3} \alpha_{12}^{2} \beta_{6}^{2}+a_{4} \alpha_{12}\left(\beta_{6}^{2}\right)^{2} \\
& +a_{5}\left(\beta_{6}^{2}\right)^{3}+a_{6} \beta_{6} \gamma_{18} \alpha_{12}+a_{7} \beta_{6} \gamma_{18} \beta_{6}^{2},
\end{aligned}
$$

using some $a_{i} \in \mathbb{R}(i=1,2, \ldots, 7)$, where $\alpha_{12}=a\left(a^{3}+8 p^{3}\right)$, $\beta_{6}=a^{2}-12 b, \gamma_{18}=a^{6}-20 a^{3} p^{3}-8 p^{6}$, and $\delta_{36}=p^{3}\left(a^{3}-p^{3}\right)^{3}$ and $a=x^{3}+y^{3}+z^{3}, p=3 x y z$, and $b=x^{3} y^{3}+x^{3} z^{3}+y^{3} z^{3}$. From the minimum weight, we have the following:

$$
\begin{aligned}
a_{2}=\frac{3281}{13824}-\frac{a_{1}}{64}, & a_{3}=\frac{203}{4608}-\frac{9 a_{1}}{256}, \\
a_{4}=\frac{1763}{13824}+\frac{3 a_{1}}{128}, & a_{5}=-\frac{277}{13824}-\frac{a_{1}}{256}, \\
a_{6}=\frac{1133}{1728}+\frac{3 a_{1}}{64}, & a_{7}=-\frac{77}{1728}-\frac{a_{1}}{64} .
\end{aligned}
$$

Since $W_{C}(x, y, z)$ contains the term $\left(15180+2916 a_{1}\right) y^{15} z^{21}$, if $C$ is admissible, then

$$
a_{1}=-\frac{15180}{2916} \text {. }
$$

Hence, the complete weight enumerator of a ternary admissible extremal self-dual code containing $\mathbf{1}$ is uniquely determined, which is listed in (18).

$$
\begin{aligned}
& x^{36}+ y^{36}+z^{36}+78706260 x^{12} y^{12} z^{12} \\
&+ 682\left(x^{18} y^{18}+x^{18} z^{18}+y^{18} z^{18}\right) \\
&+ 7019232\left(x^{15} y^{15} z^{6}+x^{15} y^{6} z^{15}+x^{6} y^{15} z^{15}\right) \\
&+ 29172\left(x^{24} y^{6} z^{6}+x^{6} y^{24} z^{6}+x^{6} y^{6} z^{24}\right) \\
&+ 10260316\left(x^{18} y^{9} z^{9}+x^{9} y^{18} z^{9}+x^{9} y^{9} z^{18}\right) \\
&+ 37995408\left(x^{12} y^{15} z^{9}+x^{12} y^{9} z^{15}+x^{15} y^{12} z^{9}\right. \\
&\left.+x^{15} y^{9} z^{12}+x^{9} y^{12} z^{15}+x^{9} y^{15} z^{12}\right) \\
&+ \\
&+3924756\left(x^{12} y^{18} z^{6}+x^{12} y^{6} z^{18}+x^{18} y^{12} z^{6}\right. \\
&\left.+x^{18} y^{6} z^{12}+x^{6} y^{12} z^{18}+x^{6} y^{18} z^{12}\right) \\
&+6732\left(x^{24} y^{3} z^{9}+x^{24} y^{9} z^{3}+x^{3} y^{24} z^{9}\right. \\
&\left.+58 x^{9} y^{24} z^{3}+x^{9} y^{3} z^{24}\right) \\
&+170544\left(x^{15} y^{18} z^{3}+x^{15} y^{3} z^{18}+x^{18} y^{15} z^{3}\right. \\
&\left.+x^{18} y^{3} z^{15}+x^{3} y^{15} z^{18}+x^{3} y^{18} z^{15}\right) \\
&+102\left(x^{12} y^{21} z^{3}+x^{12} y^{3} z^{21}+x^{21} y^{12} z^{3}\right. \\
&\left.+x^{21} y^{3} z^{12}+x^{3} y^{12} z^{21}+x^{3} y^{21} z^{12}\right) \\
&\left.+x^{24} z^{12}+x^{24} y^{12} z^{24}+y^{24} z^{12}\right)
\end{aligned}
$$


5.3. Condition (b). Suppose that $T_{36}$ satisfies condition (b). By the Gleason theorem (see Corollary 5 in [17]), the weight enumerator of $T_{36}$ is uniquely determined as

$$
\begin{array}{rl}
1+8 & 88 y^{9}+34848 y^{12}+1432224 y^{15} \\
& +18377688 y^{18}+90482256 y^{21}+162551592 y^{24} \\
& +97883072 y^{27}+16178688 y^{30}+479232 y^{33}
\end{array}
$$

By Theorem 6 in [16] (see also Theorem 3.1 in [2]), $L_{S}\left(T_{36}\right)$ and $L_{T}\left(T_{36}\right)$ are extremal. Hence, $\min \left(A_{3}\left(T_{36}\right)\right)=3$ and $\min \left(S\left(A_{3}\left(T_{36}\right)\right)\right)=5$.

Note that a unimodular lattice $L$ contains a 3 -frame if and only if $L \cong A_{3}(C)$ for some ternary self-dual code $C$. Let $L_{36}$ be any of the five lattices given in Table 2 . Let $L_{36}^{(3)}$ be the set $\left\{\{x,-x\} \mid(x, x)=3, x \in L_{36}\right\}$. We define the simple undirected graph $\Gamma\left(L_{36}\right)$, whose set of vertices is the set of 480 pairs in $L_{36}^{(3)}$ and two vertices $\{x,-x\},\{y,-y\} \in L_{36}^{(3)}$ are adjacent if $(x, y)=0$. It follows that the 3 -frames in $L_{36}$ are precisely the 36-cliques in the graph $\Gamma\left(L_{36}\right)$. We have verified by MAGMA that $\Gamma\left(L_{36}\right)$ are regular graphs with valency 368 , and the maximum sizes of cliques in $\Gamma\left(L_{36}\right)$ are 12 . Hence, none of these lattices is constructed from some ternary self-dual code by Construction $\mathrm{A}$.

\section{Disclosure}

This work was carried out at Yamagata University.

\section{Conflict of Interests}

The author declares that there is no conflict of interests regarding the publication of this paper.

\section{Acknowledgments}

The author would like to thank Masaaki Kitazume for bringing the observation in Section 5 to the author's attention. This work is supported by JSPS KAKENHI Grant no. 23340021.

\section{References}

[1] G. Nebe and N. J. A. Sloane, "Unimodular lattices, together with a table of the best such lattices, in A Catalogue of Lattices," http://www.math.rwth-aachen.de/ Gabriele.NebeLATTICES/.

[2] P. Gaborit, "Construction of new extremal unimodular lattices," European Journal of Combinatorics, vol. 25, no. 4, pp. 549-564, 2004.

[3] M. Harada, "Construction of some unimodular lattices with long shadows," International Journal of Number Theory, vol. 7, no. 5, pp. 1345-1358, 2011.

[4] M. Harada, "Optimal self-dual $\mathbb{Z}_{4}$-codes and a unimodular lattice in dimension 41," Finite Fields and Their Applications, vol. 18, no. 3, pp. 529-536, 2012.

[5] M. Harada, "Extremal Type II $\mathbb{Z}_{k}$-codes and $k$-frames of odd unimodular lattices," To appear in IEEE Transactions on Information Theory.
[6] E. M. Rains and N. J. Sloane, "The shadow theory of modular and unimodular lattices," Journal of Number Theory, vol. 73, no. 2, pp. 359-389, 1998.

[7] J. H. Conway and N. J. Sloane, "A note on optimal unimodular lattices," Journal of Number Theory, vol. 72, no. 2, pp. 357-362, 1998.

[8] C. Parker, E. Spence, and V. D. Tonchev, "Designs with the symmetric difference property on 64 points and their groups," Journal of Combinatorial Theory Series A, vol. 67, no. 1, pp. 2343, 1994.

[9] G. Nebe and B. Venkov, "Unimodular lattices with long shadow," Journal of Number Theory, vol. 99, no. 2, pp. 307-317, 2003.

[10] W. Bosma, J. Cannon, and C. Playoust, "The Magma algebra system. I: the user language," Journal of Symbolic Computation, vol. 24, no. 3-4, pp. 235-265, 1997.

[11] J. H. Conway and N. J. A. Sloane, Sphere Packing, Lattices and Groups, Springer, New York, NY, USA, 3rd edition, 1999.

[12] J. H. Conway and N. J. Sloane, "Self-dual codes over the integers modulo 4," Journal of Combinatorial Theory Series A, vol. 62, no. 1, pp. 30-45, 1993.

[13] V. Pless, J. S. Leon, and J. Fields, "All $\mathbb{Z}_{4}$ codes of type II and length 16 are known," Journal of Combinatorial Theory Series A, vol. 78, no. 1, pp. 32-50, 1997.

[14] A. E. Brouwer, "Bounds on the size of linear codes", in Handbook of Coding Theory, V. S. Pless and W. C. Huffman, Eds., pp. 295461, Elsevier, Amsterdam, The Netherlands, 1998.

[15] D. Jungnickel and V. D. Tonchev, "Exponential number of quasisymmetric SDP designs and codes meeting the Grey-Rankin bound," Designs, Codes and Cryptography, vol. 1, no. 3, pp. 247253, 1991.

[16] M. Harada, M. Kitazume, and M. Ozeki, "Ternary code construction of unimodular lattices and self-dual codes over $\mathbb{Z}_{6}$," Journal of Algebraic Combinatorics, vol. 16, no. 2, pp. 209-223, 2002.

[17] C. L. Mallows, V. Pless, and N. J. A. Sloane, "Self-dual codes over GF(3)," SIAM Journal on Applied Mathematics, vol. 31, pp. 649$666,1976$. 


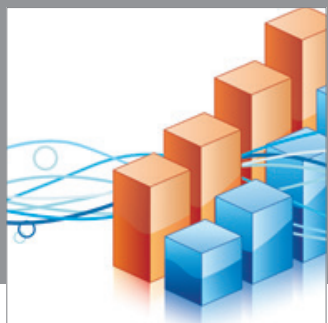

Advances in

Operations Research

mansans

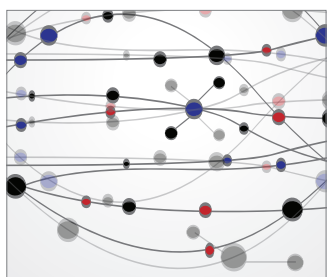

The Scientific World Journal
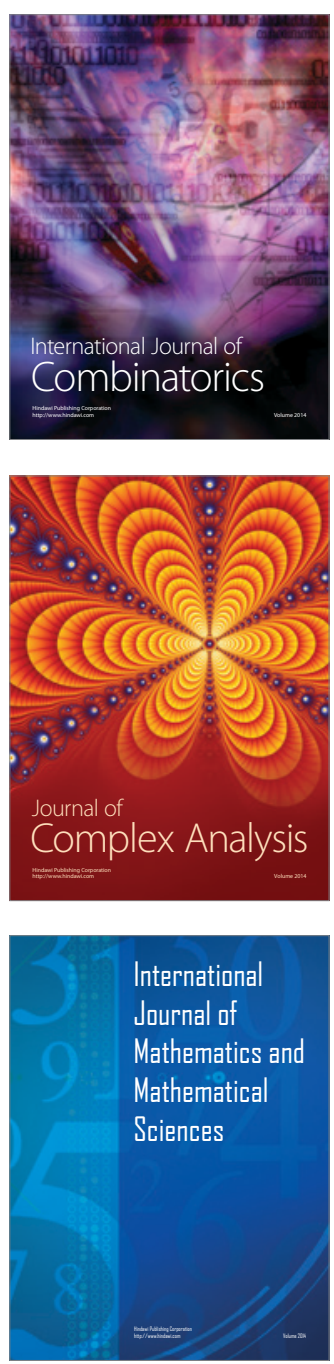
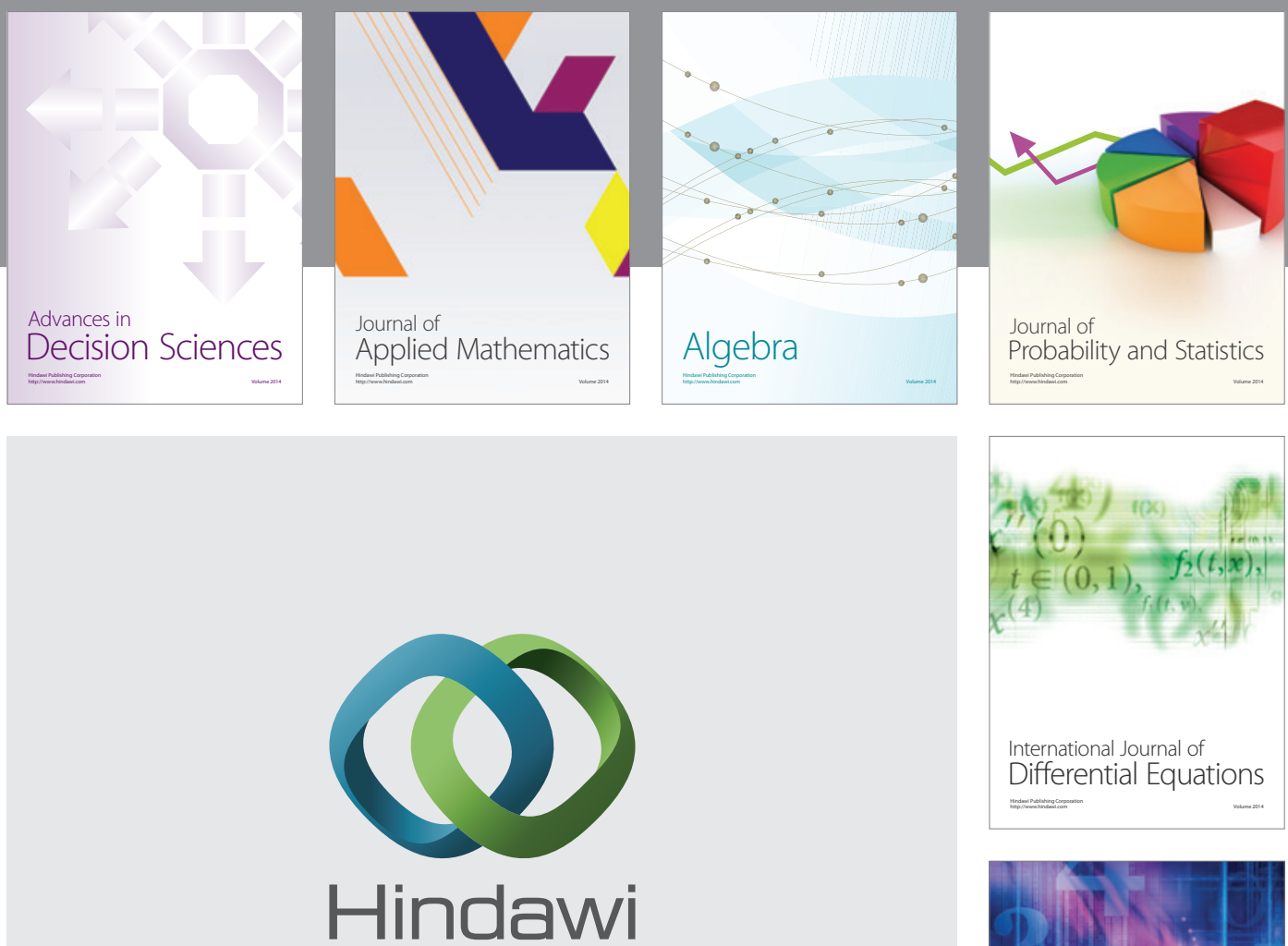

Submit your manuscripts at http://www.hindawi.com
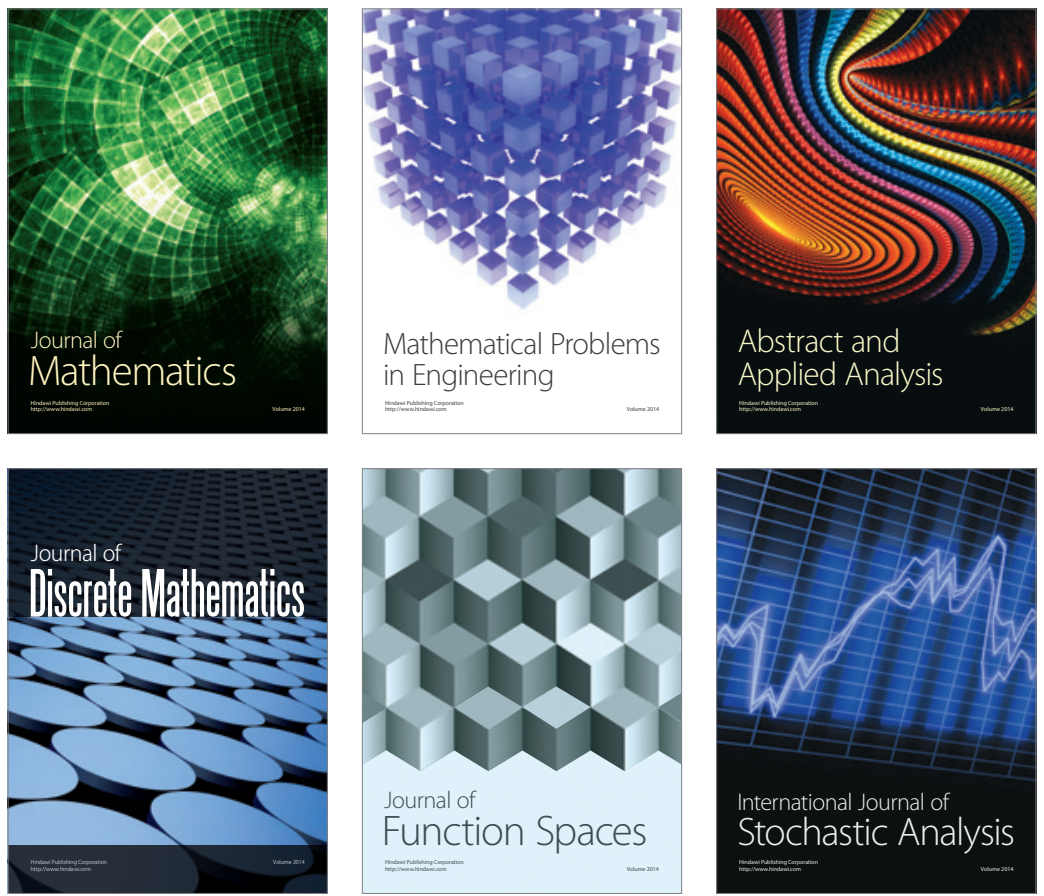

Journal of

Function Spaces

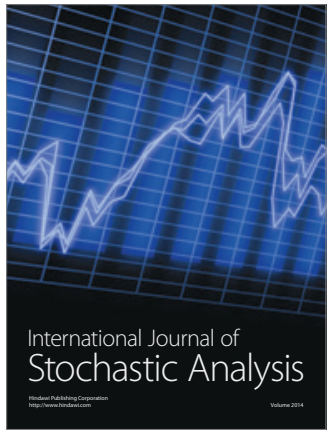

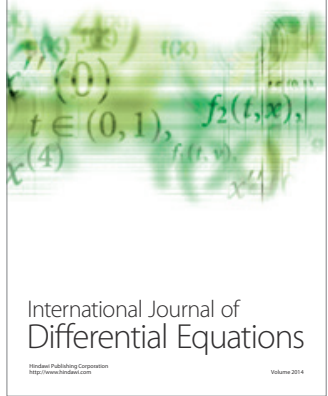
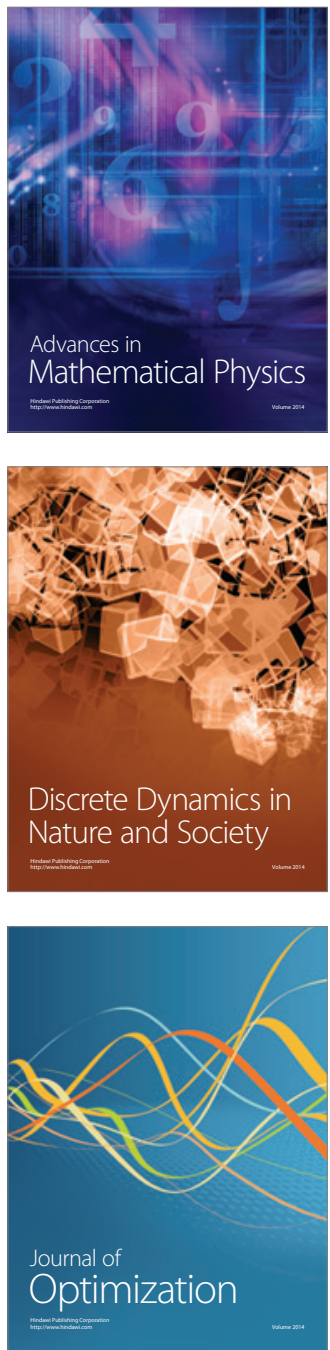